\title{
Rewarding the fruits of young labor
}

T he recipients of the first annual New York Academy of Sciences (NYAS) Blavatnik Awards for Young Scientists were honored recently at a gala dinner in New York City.

The NYAS Blavatnik Awards for Young Scientists are made possible by a grant from the Blavatnik Charitable Foundation. The awards were established to reward young scientists and engineers (born, for the 2007 awards, on or after January 1, 1965) based in the New York metropolitan region New York, New Jersey, and Connecticut who have made innovative contributions to interdisciplinary research. NYAS President Ellis Rubinstein told the JCI that the idea for the new awards stemmed from both the efforts of the NYAS to identify and support exciting young scientists, as it does through its Frontiers of Science program (1), and the desire of Russian entrepreneur Leonard Blavatnik to provide financial support to a portfolio of young scientists.

There were more than 250 applications for the inaugural awards, and these were narrowed down to 14 finalists by a panel of more than 40 prominent scientists. Each finalist was awarded $\$ 5,000$, and five of these finalists were chosen as winners, receiving an additional $\$ 20,000$. The use of the prize money is not restricted; although one of the winners, biologist Leslie Vosshall from The Rockefeller University, told the JCI that she had not yet figured out where to invest the money, she felt that the unrestricted funds would allow her group to do some risky things other funds would not.

The awards were designed to recognize endeavors in the life, physical, and social sciences as well as engineering, and this is reflected in the breadth of the research being undertaken by the 2007 finalists - from teaching computers to interpret images, to making biodegradable plastics, to understanding how the immune system detects and responds to microorganisms. However, the research conducted in the laboratories of four of the five winners, even those considered physical scientists, might have clinical applications. For example, although the work of George Malliaras from Cornell University is in organic electronics, he is working on developing handheld medical diagnostic devices. Similarly, Milan Stojanovic from Columbia University is applying his research on nucleic acidbased molecular devices to medical problems, such as leukemias and lymphomas.

Traditional biological science is conducted in the laboratory of Vosshall, who told the JCI that her interest in science began when she was a high school junior and her uncle, who was a scientist at Syracuse University, invited her to work in his summer laboratory. There she discovered that laboratory science had no resemblance to the dreary textbooks and rote memorization of high school science. Much of Vosshall's research uses the genetically tractable animal Drosophila melanogaster to understand how the brain interprets odors and how this contributes to specific behavior, but a collaborative project that she has recently begun aims to determine how olfactory signals have an impact on the host-seeking behavior of the mosquito Anopheles gambiae, a carrier of the parasite that causes malaria. The hope is that understanding this might lead to the development of a highly effective insect repellent that would help reduce the transmission of the malariacausing parasite.

Fellow winner Ruslan Medzhitov from Yale University was described as "unique" by Alexander Rudensky, Professor of Immunology at the University of Washington, Seattle, and a postdoc in immunologist Charles Janeway's laboratory before Medzhitov. Rudensky told the JCI that he doesn't know anyone in the biomedical sciences who thinks conceptually like Medzhitov, who can turn complex conceptual programs into testable hypotheses. Medzhitov's group studies how the evolutionarily conserved arm of the immune system, the innate immune system, recognizes microorganisms and how it responds to such recognition. This interest stems from his postdoctoral work in Janeway's laboratory, in which he played a central

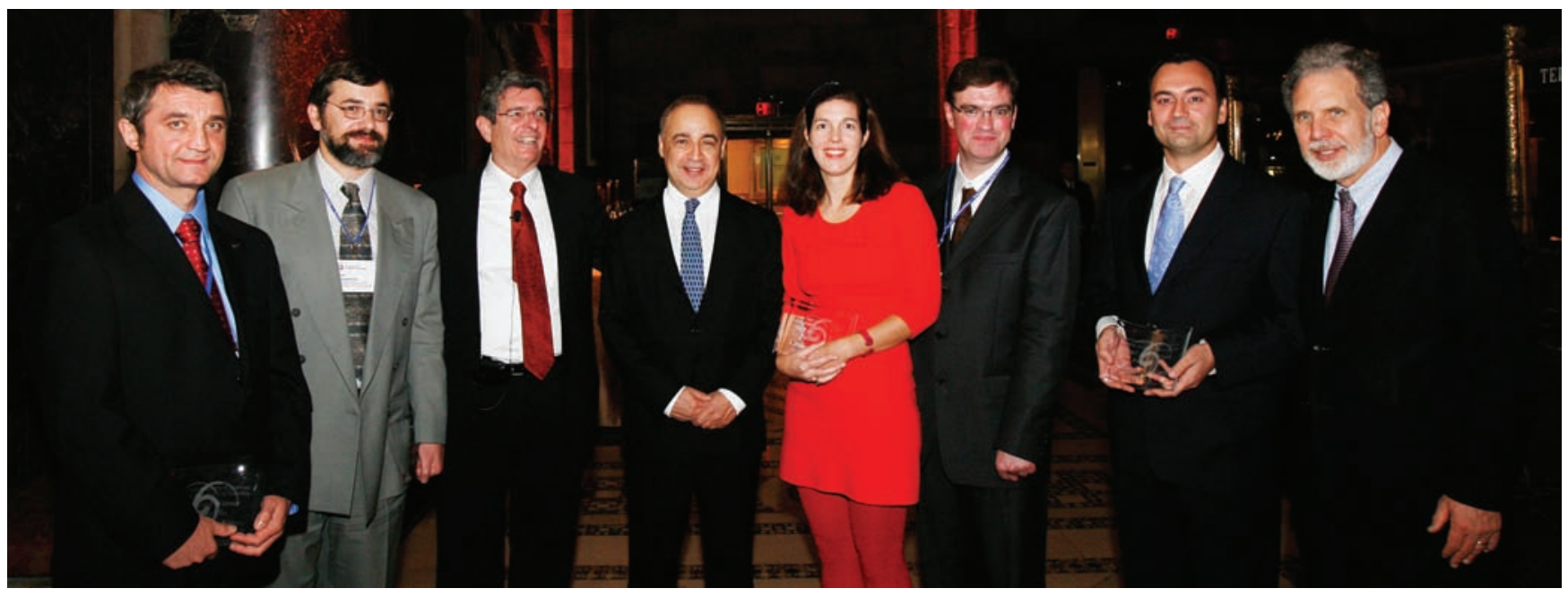

Five winners of the first annual NYAS Blavatnik Awards for Young Scientists were honored at a gala dinner in New York City. Pictured are Ruslan Medzhitov, Milan Stojanovic, Ellis Rubinstein (NYAS President), Leonard Blavatnik, Leslie Vosshall, Léon Bottou, George Malliaras, and John Sexton (Chair of the NYAS Board of Govenors). Image credit: New York Academy of Sciences. 


\title{
Winners of the New York Academy of Sciences (NYAS) Blavatnik Awards for Young Scientists
}

\author{
Léon Bottou \\ Position: Distinguished Research Scientist, NEC Labs America, Princeton, New Jersey, USA
}

George Malliaras

Position: Director, Cornell Nanoscale Science and Technology Facility, and Associate Professor of Materials Science and Engineering, Cornell University

Ruslan Medzhitov

Position: Professor, Section of Immunobiology, Yale University School of Medicine and Investigator, Howard Hughes Medical Institute

Milan Stojanovic

Position: Assistant Professor of Medical Sciences, Columbia University

Leslie Vosshall

Position: Chemers Family Associate Professor, Head of Laboratory of Neurogenetics and Behavior, The Rockefeller University

role in characterizing a family of innate immune receptors that sense microorganisms, the TLRs. These studies had such a profound effect on the field of mammalian innate immunology that Medzhitov was appointed to the faculty of Yale University in 1998 and as a Howard Hughes Medical Institute assistant investigator in 2000. Gregory Barton, an assistant professor at the University of California, Berkeley, and a postdoc in Medzhitov's lab for five years, told the JCI that "what really sets Medzhitov apart is his uncanny intuition about how biology works and his ability to implement his ideas experimentally - it is one thing to have brilliant ideas; it is another to prove them."

The 2008 NYAS Blavatnik Awards for Young Scientists (2) will also include a category for postdoctoral scientists, highlight- ing the desire of the NYAS and the Blavatnik Charitable Foundation to support scientists in the midst of their careers.

\section{Karen Honey}

1. New York Academy of Sciences. Academy programs: Frontiers of Science. http://www.nyas.org/ programs/frontiers.asp.

2. New York Academy of Sciences. About the Academy: 2008 Blavatnik Awards for Young Scientists. http://www.nyas.org/about/blavatnikawards.asp. 